Francisco Chiaravalloti Neto ${ }^{1,2}$

Maria Silvia de Moraes 3

Maria Aparecida Fernandes 2

\section{Avaliação dos resultados de atividades de incentivo à participação da comunidade no controle da dengue em um bairro periférico do Município de São José do Rio Preto, São Paulo, e da relação entre conhecimentos e práticas desta população}

\author{
Results of activities encouraging community \\ participation in dengue control in an outlying \\ neighborhood of São José do Rio Preto, \\ São Paulo, and the relationship between \\ the population's knowledge and habits
}

1 Centro Universitário de Rio Preto.

Rua Rio Negro 160 São José do Rio Preto, SP 15091-390, Brasil.

2 Superintendência de

Controle de Endemias. Av. Philadelpho M. G. Netto 3101, 3o andar,

São José do Rio Preto, SP 15060-040, Brasil.

3 Faculdade de Medicina de São José do Rio Preto. Av. Faria Lima 5416, São José do Rio Preto, SP 15090-000, Brasil.

fcneto@riopreto.com.br

\begin{abstract}
This study evaluates the results of an educational campaign developed in an outlying neighborhood of São José do Rio Preto and the relationship between the population's knowledge and habits. Prior to and after the educational activities, samples were taken to measure level of knowledge concerning dengue and its vectors, breeding sites, and control measures. Potential breeding sites were also counted. Knowledge of the disease and its vectors and breeding sites increased, but the same was not true for control measures focusing on water recipients serving as potential breeding sites. There was no change in the number of breeding sites between the first and second stages of the research. Thus, despite increased knowledge, the local population's habits did not changes. These findings are worrisome, since the main goal of the educational campaign, i.e., the change of habits concerning breeding sites for dengue vectors, has not been achieved.
\end{abstract}

Key words Dengue; Disease Vectors; Knowledge, Attitudes, Practice; Consumer Participation; Community Participation

Resumo Este estudo foi realizado com o objetivo de avaliar os resultados de um trabalho educativo desenvolvido em um bairro periférico de São José do Rio Preto e a relação existente entre conhecimentos e práticas desta população. Prévia e posteriormente às atividades educativas, foram realizadas amostragens para medir o grau de conhecimento da população sobre dengue, seus vetores, criadouros e medidas de controle, e as quantidades de criadouros potenciais existentes. Pode-se afirmar que houve ganhos de conhecimentos da população local em termos da doença, seus vetores e criadouros, mas não em termos das medidas adotadas para controle de recipientes. Com relação aos criadouros, praticamente não houve alterações em suas quantidades entre as duas fases da pesquisa. Portanto, apesar da identificação de ganhos de conhecimento, não houve mudanças das práticas da população local. Os achados desta pesquisa são preocupantes, uma vez que o principal resultado a ser alcançado pelo trabalho educativo, a mudança de práticas em relação aos criadouros dos vetores da dengue, não está sendo viabilizado.

Palabras-chave Dengue; Vetores de Doenças; Conhecimentos, Atitudes e Prática; Participação Comunitária 


\section{Introdução}

Um dos grandes problemas das doenças transmitidas por vetores é conseguir a participação efetiva da população no seu controle. No caso específico da dengue, como a grande maioria dos criadouros de Aedes aegypti e/ou Aedes albopictus encontram-se nas residências ou em suas imediações, esta questão torna-se vital para a eficácia das medidas de controle.

No Estado de São Paulo, o modelo que vem sendo adotado para o equacionamento do problema tem utilizado ferramentas como a eliminação e/ou tratamento com larvicidas de criadouros dos vetores realizados por equipes municipais e/ou estaduais, nebulização de inseticidas a ultrabaixo volume com equipamentos portáteis ou pesados para eliminação de mosquitos adultos em momentos de transmissão de dengue, tratamento perifocal com inseticidas de criadouros, principalmente nos locais com grande presença deles, como borracharias, ferros-velhos etc., e atividades incentivando a participação da comunidade. Mesmo com a adoção dessas medidas, anualmente tem ocorrido transmissão de dengue em vários municípios do estado (Secretaria de Estado de Saúde, dados não publicados).

O incentivo à participação comunitária têm se utilizado de recursos como veiculação de mensagens pela mídia, atividades com multiplicadores, reuniões em sociedades de amigos de bairros, igrejas e creches; uso de outdoors, faixas, painéis, cartazes, folhetos e balões; palestras em escolas, feiras educativas etc. No Município de São José do Rio Preto, objeto deste estudo, tais atividades vem sendo realizadas desde 1985.

A principal característica desses trabalhos educativos é a definição de um nível de conhecimento como ideal e a tentativa de passá-lo para a população, supondo que, uma vez adquirido, implicará mudanças de hábito, isto é, haverá reflexos no domicílio, com a eliminação ou pelo menos a diminuição do número de recipientes que possam criar os vetores da dengue. Essas atividades não levam em conta os conhecimentos prévios e a visão que a população tem sobre o problema, nem incentivam a sua participação na elaboração das propostas educativas. Outra característica é a ausência de discussão com a população sobre suas prioridades.

Trabalhos já realizados têm mostrado que este conhecimento ideal pode até ser incorporado pela população, mas não necessariamente corresponderá a uma mudança de hábitos, ou seja, não ocorrerá, pelo menos na intensi- dade desejada, uma diminuição do número de criadouros dos vetores a ponto de evitar a ocorrência de transmissão de dengue (Chiaravalloti Neto, 1997a; Gordon, 1988; Winch et al., 1991).

Tendo como base a idéia de que a aquisição de informação não significa necessariamente mudança de hábitos, foi realizada esta pesquisa, com o objetivo de verificar o alcance de um trabalho educativo realizado em um bairro da periferia do Município de São José do Rio Preto, São Paulo, e a relação existente entre conhecimentos e práticas da população local.

\section{Metodologia}

O Município de São José do Rio Preto situa-se a Noroeste do Estado de São Paulo e, em 1996, possuía, segundo o IBGE (1997), uma população de 326.315 habitantes. A colonização do local pelo Aedes aegypti foi detectada em 1985 (Chiaravalloti Neto, 1997b), e desde 1991 têm ocorrido epidemias de dengue (Secretaria de Estado da Saúde, dados não publicados). O bairro selecionado para o trabalho, São Deocleciano, situa-se na periferia da cidade, tratando-se um conjunto habitacional construído pelo poder público.

A pesquisa contou com duas fases, tendo a primeira ocorrido antes da realização do trabalho educativo no bairro e a segunda, após a sua realização. Em ambas as fases, foi aplicado um questionário e realizada uma contagem dos criadouros potenciais existentes em amostras de 250 domicílios. Em cada domicílio, entrevistou-se a mulher responsável pelos cuidados da casa. Por causa do seu maior conhecimento e responsabilidade sobre questões de saúde e sobre o controle doméstico, as mulheres devem ser as escolhidas para esse tipo de entrevistas (Winch et al., 1991).

O questionário aplicado foi pré-testado e contava com perguntas sobre dengue, seus vetores e medidas de controle doméstico de criadouros. A aplicação dos questionários foi realizada por estudantes de Ciências Biológicas. Os estudantes foram treinados sobre o assunto em questão e sobre técnicas de aplicação de questionários, e antes do início dos trabalhos, a título de treinamento, houve aplicação prévia do instrumento em uma pequena amostra de uma população de um bairro próximo ao selecionado. A aplicação dos questionários foi acompanhada de supervisão, realizada pelos autores, em $5 \%$ das entrevistas.

A contagem de criadouros potenciais foi realizada por três funcionários da área de pesquisa da Superintendência de Controle de En- 
demias (Sucen), também em duas fases. Foram considerados como potenciais aqueles recipientes que, contendo ou não água, poderiam vir a criar os vetores da dengue. Os criadouros pesquisados foram divididos em pneus; recipientes para armazenamento de água; vasos com água e pratos de plantas; bebedouros de animais; latas, potes, frascos e copos; recipientes fixos e outros recipientes (garrafas, vidros etc.). Não houve contagem de recipientes com larvas dos vetores, uma vez que haveria duas medidas em épocas diferentes do ano, não comparáveis em razão da sazonalidade dos mosquitos.

Para a obtenção das amostras de domicílios, o passo inicial foi o levantamento do número de quadras e casas do bairro, identificando-se um total de 49 quadras com 1.304 residências. Para cada uma das quadras, levantouse o total de casas existentes e desenhou-se um mapa, atribuindo-se um número para cada quadra. Foi elaborada uma listagem contendo todas as quadras com os respectivos números de casas, e o número de casas acumulado.

A amostra de domicílios para a primeira fase da pesquisa foi obtida da listagem supracitada, a partir do sorteio sistemático de 25 quadras, tendo cada uma participado do sorteio com peso proporcional ao seu tamanho, isto é, a probabilidade de a quadra ser sorteada era proporcional a seu número de casas. Cada uma das 25 quadras sorteadas foi visitada para a realização de um mapa de casas. De posse destes mapas, foi realizado um sorteio de dez casas em cada quadra, no sentido de se trabalhar com uma amostra de 250 domicílios. Portanto, trabalhou-se com uma amostra por conglomerados em dois estágios (quadra e domicílios), com partição proporcional ao tamanho (Kish, 1967).

Elaborou-se um impresso para ser utilizado durante a pesquisa, no qual, para cada uma das quadras escolhidas, eram registrados os endereços das dez casas a serem pesquisadas. Inicialmente, realizou-se a contagem de criadouros para evitar alguma possível alteração nessa realidade provocada pela aplicação dos questionários, e os funcionários da Sucen visitaram os domicílios de posse dos impressos com as quadras a serem trabalhadas. A primeira visita foi realizada durante a semana; as casas fechadas, vazias e com recusas foram visitadas novamente nos fins de semana por mais duas vezes. As casas vazias e as com recusas, num total de sete nesta fase, após três tentativas de visitas, foram substituídas por outras casas nas mesmas quadras. Esta etapa durou de junho a julho de 1996 .
Após a realização da pesquisa de criadouros, e de posse dos endereços das casas visitadas, procederam-se as visitas para aplicação dos questionários. Os estudantes, com os impressos contendo as quadras a serem trabalhadas, visitaram os domicílios em duplas, entrevistando sempre as mulheres responsáveis pelos mesmos. As casas fechadas ou aquelas nas quais a mulher responsável não se encontrava presente foram visitadas pelo menos mais duas vezes nos fins de semana. A não-existência de novas casas vazias e com recusas deveu-se ao fato de as mesmas terem sido substituídas durante a contagem de criadouros. Esta etapa durou de agosto a setembro de 1996.

Terminada a primeira fase da pesquisa, iniciou-se o trabalho educativo no bairro durante o mês de outubro e a primeira quinzena de novembro de 1996. Essa atividade contou com a distribuição de folhetos nas casas, afixação de cartazes em locais de utilidade pública e comerciais, utilização de dois balões de gás e afixação de faixas com dizeres sobre a dengue, estas em vários locais do bairro, reuniões com mães nas creches, palestras nas escolas, tendo como público-alvo as crianças e adolescentes, distribuição de areia nas escolas para que as crianças e os adolescentes pudessem levar para casa para colocação em vasos, instalação de feira educativa em uma das escolas do bairro, realização de reuniões com as donas de casa no Centro Comunitário e nas duas escolas do local. Paralelamente, uma campanha realizada pela mídia estava em andamento, com divulgação de mensagens pela televisão, rádios e jornais.

Terminado o trabalho educativo, procedeuse ao sorteio de uma nova amostra, também de 250 casas, para a segunda fase da pesquisa. Nesta segunda fase, seguiu-se a mesma metodologia descrita, apenas com a inversão das etapas, isto é, primeiramente a aplicação dos questionários e depois a contagem de criadouros, pois os alunos só estariam disponíveis até dezembro de 1996. Impressos com as quadras e as casas a serem pesquisadas foram distribuídos aos estudantes para a realização das entrevistas, prevendo-se três repasses e troca das casas vazias e com recusas. Na segunda fase, foram substituídas cinco casas nessa situação. Essa atividade realizou-se na segunda quinzena de novembro e durante dezembro de 1996, após o que realizou-se a contagem de criadouros no período de janeiro a fevereiro de 1997.

Os questionários da fase 1 e 2 foram tabulados e construiu-se, para cada fase, um banco de dados Dbase III (Ashton-Tate, 1985) com registro das respostas dadas e das quantidades 
de criadouros existentes por tipo. Utilizandose o programa Analysis do Epi-info 6.0 (Dean et al., 1994), as respostas dadas às várias perguntas abertas foram codificadas. Com o programa Csample do Epi-info 6.0, calcularam-se as proporções de cada resposta para ambas as fases da pesquisa, com os respectivos erros padrões (Dean et al., 1994). Este programa apresenta os resultados levando em conta o tipo de desenho amostral, no caso, conglomerados em dois estágios. Fazendo uso deste mesmo programa, calcularam-se os números médios de criadouros por tipo para as duas fases da pesquisa, com os respectivos erros padrões. Obteve-se também, para cada tipo de criadouro, a proporção de domicílios com pelo menos um criadouro e de domicílios sem criadouros, com os respectivos erros padrões.

De posse das proporções e médias e dos respectivos erros padrões, esses valores foram comparados para as duas fases da pesquisa, utilizando-se o teste estatístico para proporções (unicaudal), com aproximação pela curva normal, e o teste t-Student para as médias, e as diferenças foram consideradas estatisticamente significativas para valores de $p<0,05$. Esses cálculos foram realizados através do programa Instat 2.0 (Johnson, 1990).

Para a segunda fase da pesquisa, testou-se a existência de associação entre respostas dadas a perguntas sobre medidas de controle de criadouros e existência ou não de criadouros, utilizando-se o teste do Qui-Quadrado. Foram considerados estatisticamente significativos os valores de Qui-Quadrado correspondentes a valores de $\mathrm{p}<0,05$. Esses resultados foram calculados utilizando-se o programa Statcalc do Epi-info 6.0 (Dean et al., 1994).

\section{Resultados}

Inicialmente, na Tabela 1, são apresentadas as freqüências relativas em porcentagens de alguns tipos de respostas a perguntas sobre a doença, a forma de transmissão, o vetor e seus criadouros para as fases 1 e 2 da pesquisa, bem como o resultado do teste estatístico t-Student na comparação das proporções.

Nota-se que houve ganho de conhecimento estatisticamente significativo entre as fases $1 \mathrm{e}$ 2 em todas as perguntas, com exceção daquela sobre dengue hemorrágico. Deve-se destacar a existência de confusão entre a doença e o mosquito, pois $44 \%$ das mulheres entrevistadas afirmaram ser dengue um mosquito.

Considerando-se apenas a segunda fase da pesquisa, algumas perguntas atingiram níveis de respostas corretas que variavam de satisfatórios a bastante satisfatórios, como aquelas sobre os sintomas da dengue $(94,2 \%$ das mulheres conheciam pelo menos um sintoma),

Tabela 1

Mulheres entrevistadas em duas amostras de domicílios segundo tipo de resposta a perguntas sobre dengue e seus vetores, São José do Rio Preto, 1996

\begin{tabular}{|c|c|c|c|c|}
\hline Perguntas e respostas & Fase $1(\%)$ & Fase $2(\%)$ & Teste estatístico* & Valor de $p$ \\
\hline \multicolumn{5}{|l|}{ O que é dengue? } \\
\hline Doença transmitida por mosquito & 14,8 & 29,2 & $\mathrm{~S}$ & $<0,05$ \\
\hline Mosquito & 44,0 & 44,4 & NS & $>0,05$ \\
\hline Doença & 26,0 & 20,0 & NS & $>0,05$ \\
\hline \multicolumn{5}{|l|}{ Quais são os sintomas da dengue? } \\
\hline Três ou mais sintomas citados & 50,0 & 73,2 & $\mathrm{~S}$ & $<0,05$ \\
\hline Um ou dois sintomas citados & 40,4 & 21,2 & $\mathrm{~S}$ & $<0,05$ \\
\hline \multicolumn{5}{|l|}{ Como uma pessoa pega dengue? } \\
\hline Pela picada do mosquito & 60,0 & 78,0 & $\mathrm{~S}$ & $<0,05$ \\
\hline \multicolumn{5}{|l|}{ O que é dengue hemorrágica? } \\
\hline Dengue pela 2 a vez, mais forte ou mais grave & 14,4 & 9,2 & NS & $>0,05$ \\
\hline \multicolumn{5}{|l|}{ Como é o mosquito da dengue? } \\
\hline Pernilongo ou mosquito rajado & 9,2 & 14,8 & $\mathrm{~S}$ & $<0,05$ \\
\hline Pernilongo & 36,4 & 29,2 & NS & $>0,05$ \\
\hline \multicolumn{5}{|l|}{ Criadouros do mosquito? } \\
\hline Três ou mais criadouros citados & 13,6 & 30,0 & $\mathrm{~S}$ & $<0,05$ \\
\hline Um ou dois criadouros citados & 40,4 & 21,6 & $\mathrm{~S}$ & $<0,05$ \\
\hline
\end{tabular}

* NS = não significatico; $S$ = signicativo. 
sobre como uma pessoa pega a doença e sobre os criadouros do mosquito $(51,6 \%$ conheciam pelo menos um criadouro). Por outro lado, os conhecimentos sobre dengue hemorrágica são muito incipientes.

Na Tabela 2, são apresentadas as proporções de respostas corretas a perguntas sobre medidas para evitar o mosquito da dengue em vários tipos de criadouros. Não houve nenhum ganho de conhecimento estatisticamente significativo, notando-se que as proporções de respostas corretas são pelo menos satisfatórias.

Observa-se que os conhecimentos prévios ao trabalho educativo sobre algumas das questões levantadas apresentaram níveis também satisfatórios, muito provavelmente em função do desenvolvimento de atividades educativas no Município desde 1985.
Na Tabela 3, têm-se as proporções de domicílios com um ou mais criadouros nas duas fases da pesquisa. Com exceção de latas, potes, frascos e copos, não houve diminuição estatisticamente significativa dessas proporções para os demais.

Na Tabela 4, são apresentados os números médios de criadouros (por tipos) por domicílios para as duas fases do trabalho. Para nenhum dos tipos houve diminuição estatisticamente significativa dos números médios. Nota-se que, no local, os principais criadouros são os vasos com água e pratos de xaxim; as latas, potes, frascos e copos; os bebedouros e outros recipientes (não classificáveis nas demais categorias, como garrafas e vidros, por exemplo).

Na Tabela 5, e para a segunda fase da pesquisa, estão alguns cruzamentos entre as respostas

Tabela 2

Mulheres entrevistadas em duas amostras de domicílios segundo tipo de respostas a perguntas sobre o que fazer para evitar o mosquito da dengue em vários tipos de criadouros, São José do Rio Preto, 1996.

\begin{tabular}{lccc}
\hline Perguntas e respostas & Fase 1 (\%) & Fase 2 (\%) & Teste estatístico* Valor de p \\
\hline $\begin{array}{l}\text { Pneus? } \\
\quad \text { Respostas corretas }\end{array}$ & 55,6 & 55,6 & NS \\
$\begin{array}{l}\text { Vasos com água e pratos de plantas? } \\
\quad \text { Respostas corretas }\end{array}$ & 66,4 & 68,0 & NS \\
$\begin{array}{l}\text { Garrafas e vidros? } \\
\quad \text { Respostas corretas }\end{array}$ & 82,4 & 80,4 & NS \\
$\begin{array}{l}\text { Latas? } \\
\quad \text { Respostas corretas }\end{array}$ & & & $>0,05$ \\
$\quad \begin{array}{l}\text { Caixas d'água, tanques, barris e tambores } \\
\quad \text { Respostas corretas }\end{array}$ & 71,6 & 72,0 & NS \\
\hline
\end{tabular}

* NS = não significativo.

Tabela 3

Domícilios de duas amostras segundo tipo e existência de criadouros potenciais do mosquito da dengue, São José do Rio Preto, 1996-1997.

\begin{tabular}{|c|c|c|c|c|}
\hline \multirow[t]{2}{*}{ Tipos de criadouros } & \multicolumn{2}{|c|}{$\begin{array}{l}\text { Domicílios com } 1 \text { ou } \\
\text { mais criadouros (\%) }\end{array}$} & \multirow[t]{2}{*}{ Teste estatístico* } & \multirow[t]{2}{*}{ Valor de $p$} \\
\hline & Fase 1 & Fase 2 & & \\
\hline Pneus & 6,4 & 5,2 & NS & $>0,05$ \\
\hline Recipientes para armazenamento de água & 3,6 & 1,2 & NS & $>0,05$ \\
\hline Vasos com água e pratos de plantas & 85,2 & 79,2 & NS & $>0,05$ \\
\hline Bebedouros de animais & 53,6 & 55,2 & NS & $>0,05$ \\
\hline Latas, potes, frascos e copos & 50,6 & 34,4 & $\mathrm{~S}$ & $<0,05$ \\
\hline Recipientes fixos & 1,6 & 3,2 & NS & $>0,05$ \\
\hline Outros recipientes & 29,6 & 32,8 & NS & $>0,05$ \\
\hline
\end{tabular}

* NS = não significativo; $S$ = significativo. 
Tabela 4

Domicílios de duas amostras segundo tipo e número médio de criadouros potenciais do mosquito da dengue, São José do Rio preto, 1996-1997.

\begin{tabular}{lcccc}
\hline Tipos de criadouros & \multicolumn{2}{c}{ no médio de criadouros } & Teste estatístico* & Valor de $\mathbf{p}$ \\
& Fase 1 & Fase 2 & & \\
\hline Pneus & 0,09 & 0,08 & NS & $>0,05$ \\
Recipientes para armazenamento de água & 0,04 & 0,02 & NS & $>0,05$ \\
Vasos com água e pratos de plantas & 5,44 & 5,27 & NS & $>0,05$ \\
Bebedouros de animais & 0,93 & 0,76 & NS & $>0,05$ \\
Latas, potes, frascos e copos & 3,72 & 2,14 & NS & $>0,05$ \\
Recipientes fixos & 0,02 & 0,07 & NS & $>0,05$ \\
Outros recipientes & 1,42 & 1,89 & NS & $>0,05$ \\
Total & 11,71 & 10,23 & NS & $>0,05$ \\
\hline
\end{tabular}

* NS = não significativo.

Tabela 5

Domicílios de uma amostra (fase 2) segundo tipo de resposta a perguntas sobre o que fazer para evitar o mosquito da dengue em vários tipos de criadouros e a existência do tipo de criadouro, São José do Rio Preto, 1996-1997.

\begin{tabular}{|c|c|c|c|c|c|c|}
\hline \multirow[t]{3}{*}{ Perguntas e respostas } & \multicolumn{4}{|c|}{ Existência do tipo de criadouro } & \multirow[t]{3}{*}{ Teste do $\chi^{2 *}$} & \multirow[t]{3}{*}{ Valor de $p$} \\
\hline & & & & & & \\
\hline & $\mathrm{n}$ & $\%$ & $\mathrm{n}$ & $\%$ & & \\
\hline \multicolumn{7}{|c|}{ Vasos com água e pratos de plantas? } \\
\hline Resposta correta & 34 & 20,0 & 136 & 80,0 & & \\
\hline Resposta incorreta & 18 & 22,5 & 62 & 77,5 & NS & $>0,05$ \\
\hline \multicolumn{7}{|l|}{ Garrafas e vidros? } \\
\hline Resposta correta & 121 & 68,0 & 57 & 32,0 & & \\
\hline Resposta incorreta & 47 & 65,3 & 25 & 34,7 & NS & $>0,05$ \\
\hline \multicolumn{7}{|l|}{ Latas? } \\
\hline Resposta correta & 63 & 64,3 & 35 & 35,7 & & \\
\hline Resposta incorreta & 101 & 66,4 & 51 & 33,6 & NS & $>0,05$ \\
\hline
\end{tabular}

* NS = não significativo.

dadas pelas mulheres a perguntas sobre as formas de eliminação dos criadouros, com a existência ou não dos criadouros nos respectivos domicílios. Para as três situações analisadas não houve associação estatisticamente significativa entre o tipo de respostas (corretas ou incorretas) e a existência ou não dos criadouros, mostrando que o conhecimento da forma de controle não necessariamente implica a sua eliminação.

\section{Discussão}

Uma das alternativas viáveis para o controle da dengue é a participação efetiva da comunidade na eliminação dos criadouros do vetor. Segundo Leontisini et al. (1993), ela apresenta, além do incentivo à comunidade para resolu- ção de seus problemas, uma vantagem econômica, porque parte dos seus custos são assumidos pela própria comunidade. Na realidade atual, onde impera uma falta constante de verbas para o setor de saúde, isto pode ser um diferencial importante.

Apesar de suas vantagens, deve-se pensar na participação da comunidade como uma das medidas de controle disponíveis e associá-la a outras atividades, como a redução de criadouros, a existência de uma vigilância epidemiológica ágil que detecte rapidamente os novos casos de dengue e a existência e aplicação de legislação compatível que coíba a existência de criadouros do vetor, principalmente nos locais com grandes quantidades deles.

Alguns ensaios têm sido realizados utilizando o envolvimento da população no planeja- 
mento e/ou implementação de programas de controle da dengue com o objetivo de avaliar o grau de conhecimentos adquiridos pela população sobre a doença, seus vetores, medidas de controle e as práticas desenvolvidas para a eliminação dos criadouros. As principais conclusões a que se tem chegado são as de que, em geral, após o desenvolvimento dessas atividades, ocorre aumento do grau de conhecimentos, atingindo às vezes níveis desde satisfatórios a bastante satisfatórios. Mas, por outro lado, a aquisição dos conhecimentos não tem implicado a mudança de práticas da população em relação à eliminação de criadouros, ou esta mudança não tem sido suficiente para evitar a transmissão de dengue e dengue hemorrágico (Ayyamani et al., 1986; Swaddiwudhipong et al., 1992; Gratz, 1993; Leontisini et al., 1993; Rosenbaum et al., 1995).

Em alguns desses ensaios, verificam-se discrepâncias entre conhecimentos e práticas, isto é, a existência do conhecimento não implica necessariamente mudanças de práticas (Ayyamani et al., 1986; Rosenbaum et al., 1995). No ensaio realizado por Rosenbaum et al. (1995), não houve relação entre os moradores que apontaram corretamente as medidas de controle a serem adotadas e aqueles que tiveram menor número de recipientes em seus domicílios, realidade também detectada neste estudo.

Os achados desta pesquisa, semelhantes aos de outros artigos, são bastante preocupantes, uma vez que o principal resultado a ser alcançado por meio das atividades de participação comunitária, as mudanças de práticas com relação aos criadouros dos vetores da dengue, não está sendo viabilizado ou não tem atingido níveis suficientes para a não-ocorrência de transmissão da doença. Mesmo em programas de participação comunitária bem estruturados, com altos graus de comprometimento da população, as mudanças de hábitos, com reflexos importantes nos níveis de infestação dos vetores, são demoradas e nada fáceis de serem alcançadas (Gubler \& Clark, 1994).

Vários fatores podem ser apontados como responsáveis pela não-participação efetiva da população no controle de criadouros. Nathan (1993) coloca entre esses motivos a confiança exagerada, por parte das autoridades governamentais, nas rotinas de aplicação de larvicidas no estilo da erradicação e o papel passivo das comunidades na condução do problema. Para Clark (1995), o interesse reduzido da comunidade em participar de atividades de controle tem como razões a desconfiança, por parte da população, nos representantes governamentais; a existência de crenças colocando dengue como um acontecimento inevitável e também a apatia das comunidades. Ayyamani et al. (1986) identificam que a maior parte do conhecimento disponível pelas comunidades é ou foi adquirido por intermédio da mídia de massa, e que este tipo de educação é geralmente inefetivo para gerar mudanças em hábitos arraigados.

Avaliando o baixo grau de participação das comunidades no controle dos vetores da dengue, Winch et al. (1994) levantam uma hipótese interessante: a do impacto negativo que as campanhas centralizadas de controle de vetores podem ter sobre o poder e a autoridade das mulheres no domínio doméstico. Esse impacto negativo poderia estar relacionado à intromissão dentro do espaço doméstico pelo pessoal de controle de vetores (geralmente do sexo masculino), à reorganização desse espaço, a qual ocorre durante as visitas, e à idéia de que as doenças se originam dentro desse domínio.

Os resultados e comentários aqui apresentados devem ser utilizados para uma ampla reflexão sobre as atividades educativas desenvolvidas no Município e levar a um questionamento sobre o que deveria ser feito para se atingir maior eficácia. Provavelmente, poderiam contribuir para isto o levantamento prévio do nível de conhecimentos da população e dos principais criadouros a serem eliminados, a participação da população na elaboração das propostas educativas, o desenvolvimento de atividades de acordo com as prioridades das populações locais, entre outras (Gubler \& Clark, 1994).

Leontisini et al. (1993) dão outras sugestões importantes a respeito de como aumentar o impacto das atividades de participação comunitária sobre os índices de infestação do Aedes aegypti. Para eles, deveriam ser incorporadas atividades de reciclagem de resíduos, desenvolvimento de atividades participativas nos locais de trabalho, integração de atividades comunitárias com a de rotina do serviços governamentais, etc. O aprofundamento dessa discussão com certeza poderá gerar mudanças nos trabalhos educativos desenvolvidos, no sentido de se conseguirem melhores resultados.

\section{Conclusão}

Pode-se afirmar que as atividades educativas realizadas geraram ganhos de conhecimento entre as mulheres do local e, genericamente, para toda a população do bairro, no que se refere a questões sobre a doença, seus vetores e criadouros. As respostas corretas dadas para as medidas para eliminação de criadouros, em 
geral com altos níveis de acertos, quase não sofreram alterações entre as fases da pesquisa. As quantidades de criadouros potenciais e as proporções de domicílios com pelo menos um criadouro também não sofreram alterações entre a fase 1 e 2 da pesquisa.

Conseqüentemente, ocorreu algum ganho de conhecimento sem a correspondente mudança de práticas da população. Nota-se também que, para várias questões, os níveis de conhecimentos apresentados pelas mulheres, mesmo alguns dos obtidos na primeira fase da pesquisa, vão de satisfatórios a bastante eleva- dos, fato já notado em outra pesquisa realizada com uma amostra de mulheres do município (Chiaravalloti Neto, 1997a).

Os achados dessa pesquisa são bastante preocupantes, uma vez que o principal resultado a ser alcançado pelo trabalho educativo, a mudança de hábitos com relação aos criadouros dos vetores da dengue, não está sendo viabilizado. Outro achado preocupante é o da aparente falta de relação entre níveis de conhecimento a respeito de medidas para eliminação de criadouros e sua existência ou não nos domicílios.

\section{Agradecimentos}

Os autores agradecem a Maria Cecília Goi Porto pela orientação para a elaboração do plano de amostragem; a Neuza de Fátima Adami Santana, Marlene Cândido Gonçalves de Souza e Romeu da Silva, pelo trabalho de contagem de criadouros; a Regiane Graguim Chioderoli de Araújo e Marluce Monteiro Guirado, pela tabulação e digitação dos questionários; aos alunos do Curso de Ciências Biológicas da UNIRP, pela aplicação dos questionários, e à equipe de Educação da Sucen e da Prefeitura Municipal de São José do Rio Preto, pelo trabalho educativo desenvolvido.

\section{Referências}

ASHTON-TATE, 1985. Dbase III Plus, Version 1.0 IBM/ MSDOS. Washington: Ashton-Tate.

AYYAMANI, U. D.; YING, G. C. \& SAN, O. G., 1986. A knowledge atitude and practice (KAP) study on dengue/dengue haemorrhagic fever and the Aedes mosquitoes. Medical Journal of Malaysia, 41:108115.

CHIARAVALLOTI NETO, F., 1997a. Conhecimentos da população sobre dengue, seus vetores e medidas de controle em São José do Rio Preto, São Paulo. Cadernos de Saúde Pública, 13:447-453.

CHIARAVALLOTI NETO, F., 1997b. Descrição da colonização de Aedes aegypti na região de São José do Rio preto, São Paulo. Revista da Sociedade Brasileira de Medicina Tropical, 30:279-285. 
CLARK, G. G., 1995. Situación epidemiológica del dengue en América. Desafíos para su vigilancia y control. Salud Pública de México, 37:S5-S11.

DEAN, J. A.; COULOMBIER, D.; GRENDEL, K. A.; ARNER, T. G. \& DEAN, A. G., 1994. Epi-info, Version 6.0. Atlanta: Centers of Disease Control and Prevention.

GORDON, A. J., 1988. Mixed strategies in health education and community participation: an evaluation of dengue control in the Dominician Republic. Health and Education Research, 3:399-419.

GRATZ, N. G., 1993. Lessons of Aedes aegypti control in Thailand. Medical and Veterinary Entomology, 7:1-10.

GUBLER, D. J. \& CLARK, G. G., 1994. Communitybased integrated control of Aedes aegypti: a brief overview of current programs. American Journal of Tropical Medicine and Hygiene, 50:50-60.

IBGE (Fundação Instituto Brasileiro de Geografia e Estatística), 1997. Contagem da População. Resultados Relativos a Sexo da População e Situação da Unidade Domiciliar. Vol. 1, Rio de Janeiro: IBGE.

JOHNSON, D. F., 1990. Graphoad Instat. Graphpad Software, Version 2.00. San Diego: Rutgers University.

KISH, L. S., 1967. Survey Sampling. Washington: John Wiley \& Sons Inc.

LEONTISINI, E.; GIL, E.; KENDALL, C. \& CLARK, G. G., 1993. Effect of a community-based Aedes aegypti control programme on mosquito larval production sites in El Progresso, Honduras. Transactions of the Royal Society of Tropical Medicine and Hygiene, 87:267-271.
NATHAN, M. B., 1993. Critical review of Aedes aegypti control programs in the Caribbean and selected neighboring countries. Journal of the American Mosquito Control Association, 9:1-7.

ROSENBAUM, J.; NATHAN, M. B.; RAGOONANANSIRGH, R.; RAWLINS, S.; GAYLE. C.; CHADEE, D. D. \& LLOYD, L. S., 1995. Community participation in dengue prevention and control: a survey of knowledge, attitudes and practice in Trinidade and Tobago. American Journal of Tropical Medicine and Hygiene, 53:111-117.

SWADDIWUDHIPONG, W.; CHAOVAKIRATIPONG, C.; HGUNTRA, P.; KOONCHOTE, S.; KHUMKLAM, P. \& LERDLUKANAVONGE, P., 1992. Effect of health education on community participation in control of dengue hemorrhagic fever in an urban area of Thailand. Southeast Asian Journal of Tropical Medicine and Public Health, 23:200-206.

WINCH, P.; LLOYD, L.; GODAS, M. D. \& KENDALL, C., 1991. Beliefs about the prevention of dengue and other febrile illness in Mérida, México. Journal of Tropical Medicine and Hygiene, 94:377-387.

WINCH, P. J.; LLOYD, L. S.; HOEMEKE, L. \& LEONTISINI, E., 1994. Vector control at the household: an analysis of the impact on women. Acta Tropica, 56:327-339. 